\title{
The evolution of virulence in non-0157 Shiga toxin-producing Escherichia coli
}

\author{
Brian K. Coombes ${ }^{1}$, Matthew W. Gilmour ${ }^{2,3}$ and Chelsey D. Goodman ${ }^{2}$ \\ Department of Biochemistry and Biomedical Sciences, The Michael G. DeGroote Institute for Infectious Disease Research, McMaster University, Hamilton, ON, \\ Canada \\ 2 National Microbiology Laboratory, Public Health Agency of Canada, Winnipeg, MB, Canada \\ ${ }^{3}$ Department of Medical Microbiology and Infectious Diseases, University of Manitoba, Winnipeg, MB, Canada
}

\section{Edited by:}

Elizabeth L Hartland, The University of Melbourne, Australia

\section{Reviewed by:}

Jorge Giron, University of Florida, USA

Scott Beatson, The University of

Queensland, Australia

*Correspondence:

Brian K. Coombes, Department of Biochemistry and Biomedical

Sciences, McMaster University, HSC-4H17, Hamilton, ON, Canada L8N $3 Z 5$.

e-mail:coombes@mcmaster.ca
Shiga toxin-producing Escherichia coli (STEC) are zoonotic foodborne and waterborne pathogens that are a serious public health concern because they cause outbreaks and the potentially fatal hemolytic uremic syndrome (HUS). The most common STEC serotype associated with human disease is $\mathrm{O} 157: \mathrm{H7}$, but there is a growing recognition of over 100 non-O157 serotypes that also may result in human illness. Some of these non-O157 STEC strains cause outbreaks and severe disease such as HUS and hemorrhagic colitis, whereas others are associated with only mild diarrhea or with no human disease at all. The relative scarceness of whole genome sequence data for non-O157 STEC has limited the scientific discovery into the genetic basis of these differences in virulence. Uncovering the scope of genetic diversity and phylogeny of the non-O157 STEC through targeting sequencing of clinically relevant isolates will offer new biological insight to the pathogenic behavior of these emerging pathogens. These approaches would also enable molecular risk assessment strategies to rapidly identify and respond to emerging non-O157 STEC that pose a serious public health risk to humans.

Keywords: non-0157 STEC, pathogenesis, genetics, parallel evolution, genome sequencing

\section{NOT ALL STEC ARE CREATED EQUAL}

The first outbreak of Shiga toxin-producing Escherichia coli (STEC) was recorded in the United States in 1982 (CDC, 1982) and was caused by a clone of serotype O157:H7. A retrospective survey of older culture collections from before the 1980s indicated that this pathogen was a relative newcomer on the scene with a rare prevalence (Griffin and Tauxe, 1991). Additional outbreaks of O157:H7 STEC occurred successively in the UK, continental Europe, Africa, and New Zealand over the next decade and outbreaks associated with contaminated meat, produce and water became more commonplace. In short order, O157:H7 STEC became well-known for its ability to cause outbreaks and hemolytic uremic syndrome (HUS), a serious infection complication involving acute renal failure, hemolytic anemic, and thrombocytopenia. With the awareness of the clinical significance of O157:H7 STEC came an appreciation that non-O157 STEC serotypes were variably associated with human infections as well (Johnson et al., 2006; Bettelheim, 2007). About 150 non-O157 serotypes have now been attributed to sporadic and epidemic human infections (Gould et al., 2009), presenting with a wildly variable spectrum of disease ranging from watery or bloody diarrhea, to HUS and even death. By definition, all of these STEC contain at least one Shiga toxin gene (stx), encoding the requisite molecule giving rise to HUS complications following infection (Karmali et al., 1983). However, while some non-O157 STEC produce disease symptoms indistinguishable from O157:H7 infections it is clear that not all non-O157 STEC have the capacity to cause HUS, outbreaks, or even any human infections at all. This presents a significant dilemma for practitioners of clinical medicine and public health to identify high-risk STEC at the interfaces between humans, animals, food products, and the environment. The differences in host genetics notwithstanding, it is fair to say at this point that the genetic basis underlying the varied clinical spectrum of disease in non-O157 STEC infections remains unsolved.

The ability to identify the non-O157 STEC that pose a risk to the human population using a molecular genetic approach would be a boon to clinical medicine where the diagnosis of non-O157 is challenging at best, or worst, not attempted at all (Bettelheim, 2007). It would also represent a significant public health achievement. In fact, almost 15 years ago the World Health Organization called the rapid identification of virulent non-O157 STEC a public health priority (WHO, 1998), yet few initiatives have occurred since the call to advance this agenda.

\section{MOBILE ELEMENTS ARE A SOURCE OF GENETIC NOVELTY}

Virulence in bacterial pathogens can be attained by acquisition of mobile genetic elements such as prophages, transposons, plasmids, and genomic islands (Lawrence, 2005). One class of genomic island, called pathogenicity islands (PAI), contains virulence genes that are involved in host colonization and disease (Dobrindt et al., 2004). PAIs constitute a flexible gene pool contributing to evolution and virulence potential and can be used as a genetic signature of new and emerging pathogens. In STEC for example, the locus of enterocyte effacement (LEE) is a chromosomal PAI encoding a type III secretion system (T3SS) necessary for the attaching and effacing lesion that is characteristic of disease associated with this organism. Notably, the T3SS is a key genetic determinant of both colonization and persistence in animal reservoirs and for virulence in humans following zoonotic transmission 
(Dziva et al., 2004; Coombes, 2009). Given that ruminant animals are a natural host for STEC, such data provides persuasive arguments that the origins of STEC type III secretion might have little to do with virulence, but rather its use by modern day pathogens as a virulence factor is a convenient upshot of its selection in a different ecological setting. Levin (1996) described this evolutionary trajectory as "coincidental evolution," where the factors involved in virulence evolved for a purpose other than virulence per se. Genetic screens indicate that the substrates of the T3SS, called effectors, contribute to persistence in cattle of both O157:H7 and non-O157 STEC (Van Diemen et al., 2005), and the individual contribution of some of these effector molecules in colonization, transmission and virulence has been quantified (Wickham et al., 2007; Croxen and Finlay, 2010). Such data highlight a clinically relevant link between colonization in animals and human virulence that is influenced by the repertoire of type III secreted substrates in the associated strain. This information has important implications for public health because the persistence of type III secretion-competent strains in animal populations would contemporaneously increase the opportunities for zoonotic, foodborne, and environmental transmission of the most virulent strains to humans (Coombes, 2009).

Genome sequences of O157:H7 STEC have expanded the number of mobile genetic elements well beyond the prototypical LEE PAI (Perna et al., 2001). These include many of the O-islands (OI) that were originally defined as lineage specific segments present in O157:H7 E. coli but absent from non-pathogenic E. coli $\mathrm{K} 12$. Many of these have origins in bacteriophages that when integrated as prophages into the bacterial chromosome, endowed that STEC with new genetic information that could eventually be used to modulate colonization and infection biology in humans. The individual gene content of some of these $\mathrm{O}$-islands has been linked to the epidemic potential and disease severity associated with nonO157 STEC (Coombes et al., 2008). The precise contribution of all mobile genetic elements to STEC virulence and fitness, especially non-O157 STEC, is unknown in most cases and a comprehensive investigation of virulence determinants has not been undertaken for the non-O157 STEC.

\section{PUBLIC HEALTH PATHOGENOMICS AND THE CONCEPT OF SEROPATHOTYPE}

Recognizing that the virulence potential of non-O157 STEC was likely due to heritable genetic traits, Karmali et al. (2003) established a seropathotype classification framework that considers serotype association with prior human epidemics, HUS, and diarrhea. For example, seropathotype (SPT)-A comprises O157:H7 and O157:NM which are the most common causes of outbreaks and HUS in most countries; SPT-B strains are also associated with outbreaks and HUS, but less frequently than SPT-A; SPT-C strains are associated with sporadic HUS but not epidemics; SPT-D strains are associated with diarrhea but not with outbreaks or HUS, and SPT-E comprises multiple STEC serotypes that have never been associated with human disease. Although seropathotype memberships are based on real world clinical associations (or absence thereof) with disease, the real genetic basis behind the distribution of serotypes into a seropathotype category is not known. It can be reasonably assumed to be due to the acquisition of virulence genes and their continued maintenance as part of the flexible gene pool in a strain's genome, but the genomic landscape of non-O157 STEC spanning the various SPT classes has not yet been charted to address this hypothesis in a comprehensive manner. Although informative as an ex post facto determinant of virulence potential, the mobile nature of virulence genes exposes a limitation of SPT classification as a prognostic indicator of microbial risk. Because many virulence genes are acquired by horizontal gene transfer, it is possible (and perhaps likely) that STEC that have never been associated with human disease (i.e., SPT-E) could acquire mobile genetic elements through parallel evolution that convert them into a more virulent strain capable of human infections. In this context, identifying the genetic complement involved in human infections and understanding the complex biology of this host-pathogen interaction is a major research goal.

\section{UNDERSTANDING NON-0157 STEC VIRULENCE}

The notion of parallel evolution is well-known for pathogenic $E$. coli (Donnenberg and Whittam, 2001; Croxen and Finlay, 2010). This idea was put forward over a decade ago (Reid et al., 2000) and revisited recently following the first ever sequencing of three nonO157 STEC associated with human disease, serotypes O26, O111, and O103 (Ogura et al., 2009). These seropathotype B strains are emerging pathogens with clinical importance in many countries as a source of outbreaks and HUS complications following infection (European Commission, 2007). The genome sequences for O26-, O111-, and O103-STEC affirmed that, just as O157:H7 arose multiple times in a series of genetic steps from a pathogenic O55:H7 ancestor (Wick et al., 2005), so too have pathogenic nonO157 STEC arose from the independent acquisition of mobile genetic elements harboring common virulence genes. From what common ancestor, if any, these derive from is unknown. In these SPT-B strains, the T3SS and associated elements appear to have been constructed independently by lambdoid prophages and other integrative elements that others have previously shown to be involved in the stepwise evolution of O157:H7 (Tobe et al., 2006; Ogura et al., 2009). The stepwise acquisition of about 30 known T3SS effectors in STEC, chiefly the horizontally acquired non-LEE-encoded nle genes, are particularly interesting when considered in the context of selective forces driving their maintenance in the genome.

It is known that unlinked effectors can work in concert with one another to produce a desired effect on the host cell (Tree et al., 2009; Newton et al., 2010), or have functions that oppose host phenotypes induced by other effectors (Simovitch et al., 2010). A delivery order for effectors has also been suggested, with the translocated intimin receptor, Tir, sitting atop a delivery hierarchy needed for efficient secretion of later type III cargos (Thomas et al., 2007). Also implicit is that the positive selection of newly landed effector genes would take place in a genetic background already competent for the T3SS, since bacteria would presumably find little use for secreted cargo without the secretion machinery needed to deliver it. It is currently unclear whether there is an acquisition hierarchy for the available effector repertoire, or whether T3SS effector genes can be maintained for some time under neutral selection in the genome of strains lacking effector partners or the core T3SS itself. What dictates the "order beneath chaos" (Lawrence and Hendrickson, 
2005) of STEC genome evolution? How might evolutionary intermediates be selective, and in what environmental or animal niche does this selection take place? To get a handle on these questions, more sequence information is needed. Sequence information for additional non-O157 STEC that span the clinical divide from the worst offenders (SPT-B) to the seemingly benign (SPT-E) will be the first step in uncovering the provenance of pathogenic behavior in this emerging group of bacteria.

\section{REFERENCES}

Bettelheim, K. A. (2007). The non-O157 Shiga-toxigenic (verocytotoxigenic) Escherichia coli; under-rated pathogens. Crit. Rev. Microbiol. 33, 67-87.

CDC. (1982). Isolation of E. coli O157:H7 from sporadic cases of hemorrhagic colitis - United States. MMWR Morb. Mortal. Wkly. Rep. 31, 580-585.

Coombes, B. K. (2009). Type III secretion systems in symbiotic adaptation of pathogenic and non-pathogenic bacteria. Trends Microbiol. 17, 89-94.

Coombes, B. K., Wickham, M. E., Mascarenhas, M., Gruenheid, S., Finlay, B. B., and Karmali, M. A. (2008). Molecular analysis as an aid to assess the public health risk of non-O157 Shiga toxin-producing Escherichia coli strains. Appl. Environ. Microbiol. 74, 2153-2160.

Croxen, M. A., and Finlay, B. B. (2010). Molecular mechanisms of Escherichia coli pathogenicity. Nat. Rev. Microbiol. 8, 26-38.

Dobrindt, U., Hochhut, B., Hentschel, U., and Hacker, J. (2004). Genomic islands in pathogenic and environmental microorganisms. Nat. Rev. Microbiol. 2, 414-424.

Donnenberg, M. S., and Whittam, T. S. (2001). Pathogenesis and evolution of virulence in enteropathogenic and enterohemorrhagic Escherichia coli. J. Clin. Invest. 107, 539-548.

Dziva, F., Van Diemen, P. M., Stevens, M. P., Smith, A. J., and Wallis, T. S. (2004). Identification of Escherichia coliO157: $\mathrm{H} 7$ genes influencing colonization of the bovine gastrointestinal tract using signature-tagged mutagenesis. Microbiology 150, 3631-3645.

European Commission. (2007). Surveillance of enteric pathogens in Europe and beyond: international surveillance network for the enteric infections - Salmonella, VTEC O157 and Campylobacter. Report number: 1786/2002/EC, 1-197.

Gould, L. H., Bopp, C., Strockbine, N., Atkinson, R., Baselski, V., Body, B., Carey, R., Crandall, C., Hurd, S., Kaplan, R., Neill, M., Shea, S., Somsel, P., Tobin-D'angelo, M.,
Griffin, P. M., and Gerner-Smidt, P. (2009). Recommendations for diagnosis of shiga toxin-producing Escherichia coli infections by clinical laboratories. MMWR Recomm. Rep. 58, 1-14.

Griffin, P.M., and Tauxe, R.V. (1991). The epidemiology of infections caused by ohemorrhagic E. coli, and the associated hemolytic uremic syndrome.

Johnson, K. E., Thorpe, C. M., and Sears, C. L. (2006). The emerging clinical importance of non-O157 Shiga toxinproducing Escherichia coli. Clin. Infect. Dis. 43, 1587-1595.

Karmali, M. A., Mascarenhas, M., Shen, S., Ziebell, K., Johnson, S., Reid-Smith, R., Isaac-Renton, J., Clark, C., Rahn, K., and Kaper, J. B. (2003). Association of genomic $\mathrm{O}$ island 122 of Escherichia coli EDL 933 with verocytotoxin-producing Escherichia coli seropathotypes that are linked to epidemic and/or serious disease. J. Clin. Microbiol. 41, 4930-4940.

Karmali, M. A., Steele, B. T., Petric, M., and Lim, C. (1983). Sporadic cases of haemolytic-uraemic syndrome associated with faecal cytotoxin and cytotoxin-producing Escherichia coli in stools. Lancet 1, 619-620.

Lawrence, J. G. (2005). Common themes in the genome strategies of pathogens. Curr. Opin. Genet. Dev. 15, 584-588.

Lawrence, J. G., and Hendrickson, H. (2005). Genome evolution in bacteria: order beneath chaos. Curr. Opin. Microbiol. 8, 572-578.

Levin, B. R. (1996). The evolution and maintenance of virulence in microparasites. Emerging Infect. Dis. 2, 93-102.

Newton, H. J., Pearson, J. S., Badea, L., Kelly, M., Lucas, M., Holloway, G., Wagstaff, K. M., Dunstone, M. A., Sloan, J., Whisstock, J. C., Kaper, J. B., Robins-Browne, R. M., Jans, D. A., Frankel, G., Phillips, A. D., Coulson, B. S., and Hartland, E. L. (2010). The type III effectors NleE and NleB from enteropathogenic E. coli and OspZ from Shigella block nuclear translocation of NF-kappaB p65. PLoS Pathog. Escherichia coli O157:H7, other enterEpidemiol. Rev. 13, 60-98.

\section{ACKNOWLEDGMENTS}

Work in the Coombes and Gilmour laboratories on STEC genomics is funded by an operating grant from the Genomics Research and Development Initiative and infrastructure funding (to Brian K. Coombes) from the Canada Foundation for Innovation Leaders Opportunities Fund. Brian K. Coombes is a CIHR New Investigator and the recipient of the Early Researcher Award from the Ontario Ministry of Research and Innovation.

6, e1000898. doi: 10.1371/journal. ppat.1000898

Ogura, Y., Ooka, T., Iguchi, A., Toh, H., Asadulghani, M., Oshima, K. Kodama, T., Abe, H., Nakayama, K., Kurokawa, K., Tobe, T., Hattori, M. and Hayashi, T. (2009). Comparative genomics reveal the mechanism of the parallel evolution of O157 and nonO157 enterohemorrhagic Escherichia coli. Proc. Natl. Acad. Sci. U.S.A. 106, 17939-17944.

Perna, N. T., Plunkett, G. III, Burland, V., Mau, B., Glasner, J. D., Rose, D. J., Mayhew, G. F., Evans, P. S., Gregor, J., Kirkpatrick, H. A., Posfai, G., Hackett, J., Klink, S., Boutin, A., Shao, Y., Miller, L., Grotbeck, E. J., Davis, N. W., Lim, A., Dimalanta, E. T., Potamousis, K. D. Apodaca, J., Anantharaman, T. S., Lin, J., Yen, G., Schwartz, D. C., Welch, R. A., and Blattner, F. R. (2001). Genome sequence of enterohaemorrhagic Escherichia coli O157:H7. Nature 409, 529-533.

Reid, S. D., Herbelin, C. J., Bumbaugh, A C., Selander, R. K., and Whittam, T. S. (2000). Parallel evolution of virulence in pathogenic Escherichia coli. Nature 406, 64-67.

Simovitch, M., Sason, H., Cohen, S. Zahavi, E. E., Melamed-Book, N., Weiss, A., Aroeti, B., and Rosenshine, I. (2010). EspM inhibits pedestal formation by enterohaemorrhagic Escherichia coli and enteropathogenic E. coli and disrupts the architecture of a polarized epithelial monolayer. Cell. Microbiol. 12, 489-505.

Thomas, N. A., Deng, W., Baker, N., Puente, J., and Finlay, B. B. (2007). Hierarchical delivery of an essential host colonization factor in enteropathogenic Escherichia coli. J. Biol. Chem. 282, 29634-29645.

Tobe, T., Beatson, S.A., Taniguchi, H., Abe H., Bailey, C. M., Fivian, A., Younis, R., Matthews, S., Marches, O., Frankel, G., Hayashi, T., and Pallen, M. J. (2006). An extensive repertoire of type III secretion effectors in Escherichia col O157 and the role of lambdoid phages in their dissemination. Proc. Natl. Acad. Sci. U.S.A. 103, 14941-14946.
Tree, J. J., Wolfson, E. B., Wang, D., Roe, A. J., and Gally, D. L. (2009). Controlling injection: regulation of type III secretion in enterohaemorrhagic Escherichia coli. Trends Microbiol. 17, 361-370.

Van Diemen, P.M., Dziva, F., Stevens, M.P., and Wallis, T.S. (2005). Identification of enterohemorrhagic Escherichia coli $\mathrm{O} 26: \mathrm{H}$-genes required for intestinal colonization in calves. Infect. Immun. $73,1735-1743$.

WHO. (1998). “Zoonotic non-O157 Shiga toxin-producing Escherichia coli (STEC)," in Report of a WHO Scientific Workshop Group Meeting (Berlin: World Health Organization).

Wick, L. M., Qi, W., Lacher, D. W., and Whittam, T. S. (2005). Evolution of genomic content in the stepwise emergence of Escherichia coli O157:H7. J. Bacteriol. 187, 1783-1791.

Wickham, M. E., Brown, N. F., Boyle, E. C., Coombes, B. K., and Finlay, B. B. (2007).Virulence is positively selected by transmission success between mammalian hosts. Curr. Biol. 17, 783-788.

Conflict of Interest Statement: The authors declare that the research was conducted in the absence of any commercial or financial relationships that could be construed as a potential conflict of interest.

Received: 07 March 2011; paper pending published: 11 April 2011; accepted: 14April 2011; published online: 25 April 2011. Citation: Coombes BK, Gilmour MW and Goodman CD (2011) The evolution of virulence in non-O157 Shiga toxin-producing Escherichia coli. Front. Microbio. 2:90. doi: 10.3389/fmicb.2011.00090

This article was submitted to Frontiers in Cellular and Infection Microbiology, a specialty of Frontiers in Microbiology.

Copyright (c) 2011 Coombes, Gilmour and Goodman. This is an open-access article subject to a non-exclusive license between the authors and Frontiers Media SA, which permits use, distribution and reproduction in other forums, provided the original authors and source are credited and other Frontiers conditions are complied with. 